\title{
Diagnóstico sobre la Atención a la Diversidad Estudiantil en Danza en Educación Superior
}

\author{
Diagnosis on Attention to Student Diversity in Dance in Higher Education
}

Yeny Avila García

Facultad de Artes, Universidad Autónoma de Chihuahua, México

yavila@uach.mx

Sergio Raúl Herrera Meza

Centro Universitario CIFE, México

sergioherrera@cife.edu.mx

Fecha recepción: 08/03/2020

Páginas 27-41

Fecha aceptación: 29/05/2020

\section{Resumen.}

La inclusión en el ámbito educativo de la danza es un tema controversial y sensible que manifiesta lo complejo que resulta brindar atención a la diversidad en el ámbito dancístico. El objetivo fue realizar un diagnóstico de la percepción que tienen los estudiantes de danza sobre la diversidad para conocer qué tanta disposición existe hacia la diversidad. Participaron 453 estudiantes de danza procedentes de cuatro universidades de México. Se aplicó el Cuestionario para Evaluar la Atención a la Diversidad Estudiantil en Danza (CEADED), se comprobó su nivel de confiabilidad y consistencia interna, se llevó a cabo un análisis de las frecuencias y porcentajes, y se utilizó la prueba de Kruskal-Wallis. Los resultados muestran una fuerte tendencia a la confusión, la indecisión y el rechazo de la diversidad. En este sentido, se concluye que se requiere la disposición de todos los protagonistas del mundo dancístico, especialmente el sector más joven para empujar cambios desde abajo que permitan modificar la cultura de las universidades mexicanas hacia un desarrollo serio de las prácticas inclusivas. Además, sería interesante conocer la disposición que tiene la sociedad hacia la diversidad en danza.

Palabras clave: atención a la diversidad, danza e inclusión, danza universitaria, perfil docente, prácticas inclusivas

\begin{abstract}
.
The inclusion in the educational field of dance is a controversial and sensitive issue that shows how complex it is to pay attention to diversity in the dance field since traditionally prevail criteria of perfection and excellence for its development. The objective was to make a diagnosis of the perception that dance students have about diversity to know how much disposition there is towards diversity. The study involved 453 dance students from four universities in Mexico. The Questionnaire to Evaluate Attention to Student Diversity in Dance (CEADED) was applied, its level of reliability and internal consistency was verified, an analysis of frequencies and percentages was carried out, and the Kruskal-Wallis test was used. The results show a strong
\end{abstract}


tendency to confusion, indecision and rejection of diversity. Even when an important adherence to the traditional practice is identified, a great effort is observed to develop competences oriented to the attention of diversity. In this sense, it is concluded that the disposition of all the protagonists of the dance world is required, especially the youngest sector to push changes from below that allow modifying the culture of Mexican universities towards a serious development of inclusive practices. It would also be interesting to know the disposition that society has towards diversity in dance.

Keywords: attention to diversity, dance and inclusion, university dance, teaching profile, inclusive practices

\section{1.-Introducción.}

Hablar de inclusión, envuelve un conjunto de fenómenos históricos que marcan la desigualdad en la sociedad. En un mundo globalizado como el actual, se promueven discursos que favorecen y apoyan la inclusión desde una serie de organizaciones como la ONU y la UNESCO que inducen grandes cambios a favor de la dignidad, la igualdad, la consideración, el reconocimiento y el respeto de cada persona. Actualmente, en la educación se emplea con frecuencia el término inclusión con la meta de incluir con igualdad a todas las personas a la vida estudiantil. A esto, se une el concepto de diversidad, el cual no sólo apunta a los aspectos de procedencia, lenguas, géneros, edades, condición económica, religión y cultura, sino que representa de manera general las diferencias que existen entre los seres humanos, incluyendo las distintas condiciones biológicas (Arroyo, 2013). En la educación, el concepto de diversidad tradicionalmente se gestiona desde la teoría del déficit, pretendiendo impulsar una enseñanza niveladora en busca de mantener las diferencias ocultas y controladas, pero bajo la implementación de modelos educativos intentar justificar la exclusión educativa y social. Así, la promoción de una educación para todos, donde la diversidad muestra la realidad social supone una práctica educativa inclusiva que promueva la igualdad de oportunidades, independientemente de las características y necesidades de cada individuo. Justamente, uno de los objetivos propuestos para el desarrollo sostenible es garantizar y promover una educación inclusiva y equitativa para todos (UNESCO, 2016). Esto implica no excluir a ninguna persona del derecho de formación, por eso las discusiones en torno a la inclusión plantean la configuración de propuestas que necesariamente modifican el contexto educativo en sus aspectos epistemológicos, pedagógicos y logísticos. Para ello, las instancias educativas deben propiciar las condiciones organizativas y del entorno acordes a una cultura inclusiva para abordar la problemática de la diversidad y enfrentar los riesgos. En las universidades de México, las prácticas inclusivas han crecido de manera considerable en los últimos años, se destaca un aumento en las demandas de políticas institucionales y normativas que intentan dar una respuesta positiva y pertinente en atención a la diversidad (Cornejo, 2017). Sin embargo, la búsqueda constante del ideal de la inclusión en las universidades mexicanas, de cierto modo atropellada e impositiva, no se ha logrado consumar, ya que vienen presentando dificultades y conflictos de enseñanza que han limitado 
en gran medida el aprendizaje de los estudiantes. La cuestión es que se pretende combatir con políticas de inclusión y atención a la diversidad la discriminación y los problemas sociales, cuando en muchas ocasiones estas acciones terminan reforzando y convalidando los procesos de exclusión. Invariablemente, el encuentro con personas de características 0 capacidades diferentes en el ámbito cotidiano ha generado conflictos que revelan la existencia de un profundo desconocimiento y grande prejuicio hacia la diferencia. Aunque el tema de la inclusión en la diversidad se enfoca en condiciones muy variadas de la humanidad, la sociedad y la educación relacionados con aspectos culturales, de origen, sexo, género, condición económica y religión, entre otros, el interés de este estudio está centrado especificamente en la diversidad de habilidades y capacidades físicas que pueden presentar los estudiantes de danza, como la flexibilidad, la fuerza, la coordinación y el ritmo, entre otras cualidades que atañen a la formación tradicional.

\section{1.-Dilema entre perfección e inclusión.}

En el campo de la danza, los procesos de innovación educativa inclusiva no parecen convencer del todo. El referente pedagógico sobre la formación de bailarines desde una perspectiva tradicionista alimenta la idea de que el bailarín debe convertir su cuerpo en una máquina de perfección técnica y, desde la mirada inclusiva, el componente clave para abordar la formación profesional plantea el desarrollo de las potencialidades de la persona sin importar sus condiciones reales. Autores como Campo, Palys, Passo y Tamayo (2018), aseguran que a lo largo del proceso evolutivo de la danza se ha perseguido el talento corporal a través de hábitos repetitivos, patrones bien definidos, técnicas rigurosas y movimientos bien codificados con un alto nivel de exigencias físicas. La práctica de estos modelos ha desplazado, de cierta manera, la importancia del cuerpo y, sobre todo, antepone la ejecución técnica majestuosa al cuidado integral de las personas. Sin importar el estilo dancístico que se practique de forma profesional, el intenso y complejo entrenamiento físico puede impactar de forma negativa en la salud del individuo, sufrir bajos niveles de motivación debido al cansancio extremo, provocar lesiones irreparables y crear conductas alimentarias represivas para disminuir el peso corporal (Corrales, Mena, García \& López, 2017). Esta postura imponente y exigente que se manifiesta durante el proceso formativo impone rasgos de violencia. heridas y dolor en el cuerpo que llevan a los límites físicos y psicológicos. A modo de metáfora, Brozas-Polo y Vicente-Pedraz (2017) comparan las cualidades motrices del bailarín con el movimiento de un títere, refiriendo que la búsqueda del virtuosismo técnico del cuerpo jamás culmina, pues la marioneta representa a un ser con una cualidad física superior a la del bailarín al ser capaz de obedecer y hacer de manera inmediata cualquier movimiento, por lo tanto, es inalcanzable en la realidad. El enfrentamiento de la danza con la inclusión sitúa a los docentes y a las instituciones en desventaja para hacer frente a las dificultades y pone de manifiesto la necesidad de aceptar la imperfección como ventaja para reivindicar la importancia del cuerpo.

\section{2.-Estudios sobre inclusión y atención a la diversidad en danza.}

Existen investigaciones científicas que abordan la inclusión educativa y la atención a la diversidad en el ámbito universitario, sin embargo, los estudios sobre inclusión en la danza 
abordan de manera fundamental lo relacionado con la diversidad corporal, la inclusión de la danza en la sociedad como estrategia para sensibilizar a la humanidad, y la interacción de la danza con personas que sufren alguna discapacidad notoria. Son mínimos los estudios de diagnóstico desarrollados en el campo de la formación dancística. Se deben efectuar los cambios necesarios para solucionar la situación de exclusión educativa, comenzando por la transformación en los contenidos y las estrategias; además de buscar, proponer y utilizar enfoques pedagógicos apropiados al educando desde una concepción humanista basada en la dignidad humana, en el respeto por la vida, la solidaridad y la responsabilidad social. En este sentido, de acuerdo con Tobón (2017), el enfoque socioformativo se suma a la agenda de la educación para el desarrollo sostenible, promueve el talento humano, el trabajo colaborativo e impulsa la igualdad entre las personas, ya que, independientemente de las condiciones socioculturales, cognitivas, económicas y físicas que tengan, lo que todo proceso formativo pretende es contribuir a que las personas logren desarrollarse y alcanzar los proyectos de vida que se propongan. Si bien, entre sus líneas de investigación está el de la inclusión, en el campo de la danza aún no ha incursionado. Por ello, la naturaleza de esta investigación radica en asumir la inclusión y atención a la diversidad desde la socioformación en el ámbito educativo en danza y tiene como propósito realizar un diagnóstico de la percepción que tiene el alumnado de danza sobre la diversidad para conocer qué tanta disposición existe hacia la atención a la diversidad; entendiendo esta, como la actitud que se manifiesta hacia la inclusión y diversidad, una postura que permite escuchar y dialogar a través del trato amable, mostrar sensibilidad ante la diversidad, tener apertura a relacionarse con otras personas, no imponer, ser abiertos al cambio y capaces de romper con el pesimismo.

\section{3.-Componentes fundamentales de la atención a la diversidad en danza.}

Con base a la información recabada y en función del propósito de investigación, se establecieron los componentes fundamentales para evaluar el constructo atención a la diversidad en danza (Figura 1). En concreto, se ubicaron los elementos más relevantes que ayudan a entender la percepción que tiene el alumnado sobre la diversidad, además de conocer a) qué hábitos y características provienen del origen de la danza; b) cómo se concilia la formación dancística con el cuidado del cuerpo; c) hasta qué punto la práctica docente incide en el cuidado integral del alumnado; d) qué alternativas socioformativas pueden implementarse en la práctica dancística; e) cuáles factores facilitan o dificultan la atención a la diversidad en danza; y f) qué estrategias y herramientas didácticas pueden ayudar a reducir las lesiones en los estudiantes (Avila-García \& Veytia-Bucheli, 2019).

Figura 1.-Componentes fundamentales de la atención a la diversidad en danza.

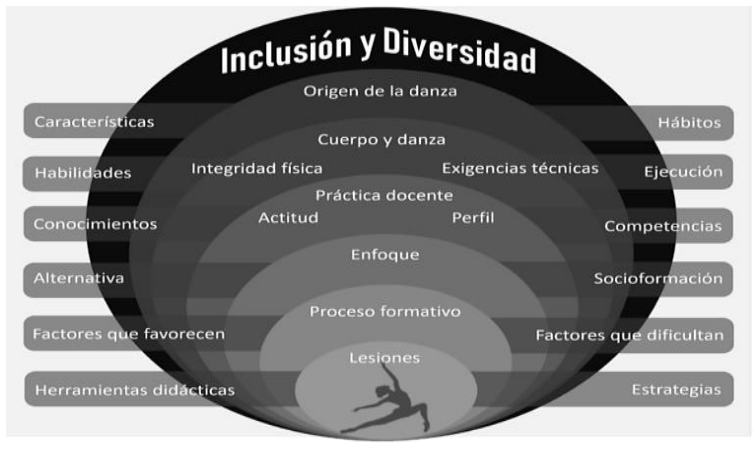




\section{Elaboración propia}

Este estudio se enfocó en las siguientes metas: 1) Realizar un diagnóstico sobre las disposiciones generales de los estudiantes de danza hacia la atención a la diversidad y la inclusión, además de evaluar las actuaciones de los docentes desde la perspectiva de los estudiantes; 2) identificar si existe alguna diferencia estadísticamente significativa en la postura de los estudiantes de las diversas áreas dancísticas (contemporánea, clásica y folklórica) ante la inclusión.

\section{2.-Metodología.}

\section{1.-Tipo de estudio.}

Se trata de un estudio con enfoque cuantitativo, diseño transversal y alcance descriptivo porque busca especificar los rasgos más peculiares o diferenciados entre la población de estudio y ofrecer un diagnóstico de la situación.

\section{2.-Instrumentos.}

Se empleó el CEADED (Cuestionario para Evaluar la Atención a la Diversidad Estudiantil en Danza), que fue diseñado y validado específicamente para el estudio (en prensa). Aporta un sistema de 43 indicadores diseñados para evaluar la atención a la diversidad dentro del marco de la inclusión educativa. Se trata de dos escalas, una primera, de opinión tipo Likert de cinco puntos, con opciones de respuesta: "Totalmente en desacuerdo/En desacuerdo/No lo sé/De acuerdo/Totalmente de acuerdo", y la segunda, de frecuencia con las opciones: "Nunca/Casi nunca/Algunas veces/Casi siempre/Siempre". Los 43 indicadores pertenecen a ocho factores: competencias docentes (10 ítems); concepción sobre diversidad (6 ítems), interés hacia la inclusión (4 ítems), estrategias didácticas (4 ítems), institución (5 ítems), competencias integradoras (4 ítems), currículo (4 ítems) y actitud docente (6 ítems).

\section{3.-Propiedades psicométricas del instrumento.}

El instrumento fue validado por juicio de expertos, obteniendo valores $V$ de Aiken superiores a 0.75 en todos los ítems, criterio mínimo contemplado para aceptar la permanencia de cada ítem dentro del instrumento. La evaluación del constructo mediante el análisis factorial exploratorio iterado con rotación Oblimin Directo resultó en una estructura de ocho factores que explican el $65.260 \%$ de la varianza con autovalores iniciales de entre 15.747 y 1.092 . En una primera etapa, el instrumento fue probado en una aplicación a un grupo piloto integrado por 50 estudiantes de danza que dio un alfa de Cronbach de 0.923 y la aplicación diagnóstica en 453 reveló confiabilidad con un alfa de Cronbach de 0.957 .

\section{4.-Participantes.}


La muestra del estudio fue conformada de manera intencional por un total de 453 estudiantes de licenciatura en danza de cuatro universidades de la República Mexicana, con edades comprendidas entre 17 y 26 años que cursaban entre el segundo y décimo semestre de la carrera durante el período enero-julio 2019, restringido a las áreas de danza contemporánea, ballet clásico y danza folklórica (Tabla 1).

Una parte de los participantes fueron invitados a través de una convocatoria directa a través de correo electrónico y redes sociales, y la otra de manera personal con el apoyo de colegas docentes y representantes de las instituciones.

Tabla 1.-Datos sociodemográficos de la muestra.

\begin{tabular}{ll}
\hline \multicolumn{1}{c}{ Características } & Datos \\
\hline $\mathrm{n}$ & 453 \\
a) Sexo & Mujeres $79 \%$ \\
& Hombres $21 \%$ \\
b) Edad & Media 20.64, d. e. \pm 2.7611 \\
c) Institución Educativa (IE) & IE1 31\% \\
& IE2 $41 \%$ \\
& IE3 $18 \%$ \\
d) Área de estudios & IE4 $10 \%$ \\
& Danza Contemporánea 47\% \\
& Danza Clásica 30\% \\
& Danza Folklórica 23\% \\
\hline
\end{tabular}

\section{Elaboración propia}

\section{5.-Procedimiento.}

a) Se aplicó el instrumento a una muestra de 453 estudiantes de danza, a través de la colaboración de docentes y directamente utilizando la modalidad de formulario electrónico y cuestionario impreso.

b) Se comprobó el nivel de confiabilidad mediante el coeficiente de alfa de Cronbach.

c) Se analizaron las frecuencias y porcentajes de respuestas en cada una de las opciones en cada uno de los ítems.

d) Se efectuó la prueba no paramétrica de Kruskal-Wallis para conocer si existen diferencias estadísticamente significativas entre las submuestras de estudiantes, conformadas por la variable área de estudios, respecto a la disposición que muestran hacia la diversidad, esto en virtud de que la variable aleatoria es ordinal, lo cual no permitiría emplear otros análisis paramétricos. Se empleó el software estadístico SSPS.

\section{6.-Consideraciones éticas.}

Previo a la aplicación del cuestionario, se hizo contacto vía correo electrónico o en persona con docentes, estudiantes y representantes de las instituciones con la intención de presentar el objetivo del estudio y resaltar que la participación era de carácter voluntario y que no se pretendía juzgar el comportamiento de las instituciones, ni de la práctica docente, y que la información proporcionada sería tratada confidencialmente, así como los resultados eran únicamente con fines de investigación y no tendrán incidencia alguna en su labor. 
A fin de guardar la confidencialidad, el cuestionario fue anónimo y los datos personales solicitados no fueron publicados ni expuestos, sólo fue analizada por el equipo investigador, esto, en cumplimiento con la Ley Federal de Protección de Datos Personales vigente en la República Mexicana y fundamentado en los principios éticos que propone el manual APA para el tratado de información derivada de instrumentos de evaluación.

\section{3.-Resultados.}

Se muestran las lecturas más relevantes en apego a los objetivos del estudio. Con la finalidad de examinar el comportamiento de las variables y confirmar la consistencia interna del instrumento y la confiabilidad de la aplicación se empleó el estadístico alfa de Cronbach (Tabla 2).

Tabla 2.-Nivel de confiabilidad.

\begin{tabular}{lcc}
\hline Factores & $\mathbf{\alpha}$ & Variables \\
\hline Competencias docentes. & .926 & 10 \\
Concepción sobre diversidad. & .899 & 6 \\
Interés hacia la inclusión. & .860 & 4 \\
Estrategias didácticas. & .858 & 4 \\
Institución & .979 & 5 \\
Competencias integradoras. & .839 & 4 \\
Currículo. & .828 & 4 \\
Actitud docente. & .882 & 6 \\
Cuestionario completo & .957 & 43 \\
\hline \multicolumn{2}{c}{ Elaboración propia }
\end{tabular}

3.1.-Frecuencia porcentual. Tendencia global hacia la diversidad.

Se procedió a realizar el análisis de las frecuencias porcentuales (\%f) registradas en cada una de las cinco opciones de respuesta por cada uno de los 43 ítems que configuran los ocho factores. Se muestran de manera general los resultados de la media de cada uno de los ítems. La valoración máxima corresponde al mayor grado de disposición hacia la inclusión desde la perspectiva de los estudiantes. En la tabla 3 , se presentan los resultados obtenidos de los factores 1,4 y 6 que ilustran desde la perspectiva del alumnado la medida con que los docentes recurren al uso de estrategias y recursos para dar respuesta a las demandas específicas de la diversidad estudiantil. 
Tabla 3.-Valoración de las competencias de los docentes desde la perspectiva de los estudiantes.

\begin{tabular}{|c|c|c|c|c|c|c|}
\hline & Ítem & Media (d.e) & $\begin{array}{l}\text { Tolime } \\
\text { Totaime } \\
\text { nte en } \\
\text { desacu } \\
\text { erdo/Nu } \\
\text { nca }\end{array}$ & $\begin{array}{l}\text { 2. En } \\
\text { desacu } \\
\text { erdo/Ca } \\
\text { si } \\
\text { nunca }\end{array}$ & $\begin{array}{l}\text { 3. No lo } \\
\text { sé/Algu } \\
\text { nas } \\
\text { veces }\end{array}$ & $\begin{array}{l}\text { 4. De } \\
\text { acuerdo } \\
\text { I } \\
\text { Casi } \\
\text { siempre }\end{array}$ \\
\hline \multirow{10}{*}{ 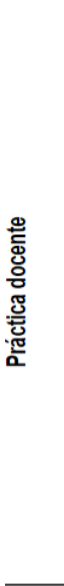 } & $\begin{array}{l}\text { 1. Los docentes desarrollan sus } \\
\text { clases de acuerdo a las } \\
\text { necesidades del alumnado }\end{array}$ & $3.68( \pm 1.37)$ & $13.7 \%$ & $8.4 \%$ & $14.6 \%$ & $30.5 \%$ \\
\hline & $\begin{array}{l}\text { 2. Los docentes refuerzan la } \\
\text { atención educativa a estudiantes } \\
\text { rezagados }\end{array}$ & $3.5( \pm 1.27)$ & $9.9 \%$ & $7.5 \%$ & $29.7 \%$ & $21.6 \%$ \\
\hline & $\begin{array}{l}\text { 3. Los docentes permiten que todos } \\
\text { expresen su opinión }\end{array}$ & $3.88( \pm 1.11)$ & $4.9 \%$ & $4.6 \%$ & $24.7 \%$ & $28.3 \%$ \\
\hline & $\begin{array}{l}\text { 4. Los docentes realizan una } \\
\text { evaluación diagnóstica previa a la } \\
\text { clase }\end{array}$ & $3.72( \pm 1.22)$ & $4.9 \%$ & $16.3 \%$ & $15.0 \%$ & $29.4 \%$ \\
\hline & $\begin{array}{l}\text { 5. Ante estudiantes con escasas } \\
\text { cualidades físicas los docentes }\end{array}$ & $3.46( \pm 1.37)$ & $13.9 \%$ & $10.2 \%$ & $22.1 \%$ & $23.6 \%$ \\
\hline & $\begin{array}{l}\text { muestran incomodidad } \\
6 \text {. Para el desarrollo de proyectos, } \\
\text { los docentes toman en cuenta a } \\
\text { todos por igual }\end{array}$ & $3.57( \pm 1.36)$ & $10.6 \%$ & $14.3 \%$ & $17 \%$ & $23.4 \%$ \\
\hline & $\begin{array}{l}\text { 7. Los docentes priorizan que se } \\
\text { cumplan al máximo los aprendizajes } \\
\text { prácticos }\end{array}$ & $3.85( \pm 1.21)$ & $6.4 \%$ & $12.1 \%$ & $7.3 \%$ & $38 \%$ \\
\hline & 8. Los docentes respetan el ritmo de & $3.60( \pm 1.15)$ & $6.4 \%$ & $10.2 \%$ & $24.7 \%$ & $33.8 \%$ \\
\hline & $\begin{array}{l}\text { 9. Los docentes crean un clima de } \\
\text { respeto y valoración por la } \\
\text { diversidad }\end{array}$ & $3.81( \pm 1.22)$ & $6.2 \%$ & $11.7 \%$ & $14.3 \%$ & $30.5 \%$ \\
\hline & $\begin{array}{l}\text { 10. Los docentes realizan prácticas } \\
\text { fuera del aula }\end{array}$ & $3.82( \pm 1.19)$ & $6.0 \%$ & $9.1 \%$ & $18.5 \%$ & $29.4 \%$ \\
\hline \multirow{4}{*}{ 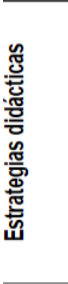 } & $\begin{array}{l}\text { 21. Los docentes utilizan diferentes } \\
\text { instrumentos de evaluación para } \\
\text { valorar el progreso de cada } \\
\text { estudiante }\end{array}$ & $3.56( \pm 1.17)$ & $4.9 \%$ & $15.7 \%$ & $23.8 \%$ & $29.8 \%$ \\
\hline & $\begin{array}{l}\text { 22. Los docentes comprenden al } \\
\text { estudiante cuando debe tomar un } \\
\text { descanso por lesión o cansancio }\end{array}$ & $3.50( \pm 1.26)$ & $6.6 \%$ & $20.3 \%$ & $15.9 \%$ & $30 \%$ \\
\hline & $\begin{array}{l}\text { 23. Los docentes evitan mensajes } \\
\text { descalificadores o situaciones } \\
\text { comparativas entre estudiantes }\end{array}$ & $3.59( \pm 1.16)$ & $4.4 \%$ & $17.4 \%$ & $17.7 \%$ & $35.1 \%$ \\
\hline & $\begin{array}{l}\text { 24. Los docentes invitan a otros } \\
\text { colegas para que observen la clase } \\
\text { y den sus comentarios }\end{array}$ & $3.72( \pm 1.24)$ & $7.7 \%$ & $10.6 \%$ & $16.6 \%$ & $31.3 \%$ \\
\hline \multirow{4}{*}{ 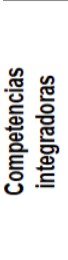 } & $\begin{array}{l}\text { 30. Los docentes valoran la opinión } \\
\text { de cada estudiante sobre su propio } \\
\text { aprendizaje }\end{array}$ & $3.68( \pm 1.20)$ & $6.8 \%$ & $9.1 \%$ & $24.7 \%$ & $27.6 \%$ \\
\hline & $\begin{array}{l}\text { 31. Los docentes integran equipos } \\
\text { con estudiantes de diferentes } \\
\text { niveles y capacidades }\end{array}$ & $3.65( \pm 1.22)$ & $5.7 \%$ & $15.9 \%$ & $16.3 \%$ & $31.3 \%$ \\
\hline & $\begin{array}{l}\text { 32. Los docentes promueven la } \\
\text { resolución de problemas a partir del }\end{array}$ & $3.77( \pm 1.14)$ & $3.5 \%$ & $12.4 \%$ & $20.5 \%$ & $30.2 \%$ \\
\hline & $\begin{array}{l}\text { 33. Los docentes utilizan un } \\
\text { vocabulario adecuado para dar las } \\
\text { instrucciones y correcciones }\end{array}$ & $3.87( \pm 1.26)$ & $7.9 \%$ & $9.5 \%$ & $10.8 \%$ & $30.5 \%$ \\
\hline
\end{tabular}

Considerando que las opciones 4 y 5 expresan alto nivel de acuerdo con la afirmación, se puede constatar que el aspecto que tiene el más alto índice de aceptación es la priorización del cumplimiento de los aprendizajes prácticos y funcionales (ítem 7: 72.2\%) y que la valoración de la diversidad (ítem 9: 67.8\%) por parte de los docentes también es una constante bastante alta, lo cual contrasta con la apreciación que tienen los encuestados acerca de la atención que los docentes dan a los estudiantes en situación especial (ítem 2: 52.9\%), que 
aunque aún es un porcentaje por encima de la mitad, es notablemente más bajo que en otros rubros. A ello se suma que en este rubro se presenta el porcentaje más alto de respuestas indefinidas (No lo sé/Algunas veces), equivalente a casi la tercera parte de los encuestados $(29.7 \%)$. Ello sugiere una tendencia notable menor que otros puntos que probablemente sean menos representativos de la sensibilidad por la inclusión por parte de los docentes, desde la apreciación de los estudiantes, como el permitir la expresión de las opiniones (ítem 3:65.8\%), las prácticas extras (ítem 10:66.5\%) y el respeto a los diferentes ritmos (ítem 8: 63.4\%). En la tabla 4, se indica la medida en cuanto a la concepción que tiene el alumnado sobre la diversidad, así como su aplicación en la danza y la postura de los docentes ante el fenómeno de inclusión.

Tabla 4.-Valoración de la postura del estudiantado hacia la inclusión y atención a la diversidad.

\begin{tabular}{|c|c|c|c|c|c|c|}
\hline & Ítem & Media (d.e) & $\begin{array}{c}1 . \\
\text { Totalme } \\
\text { nte en } \\
\text { desacu } \\
\text { erdo/Nu } \\
\text { nca } \\
\end{array}$ & $\begin{array}{c}\text { 2. En } \\
\text { desacu } \\
\text { erdo/Ca } \\
\text { si } \\
\text { nunca } \\
\end{array}$ & $\begin{array}{c}\text { 3. No lo } \\
\text { sé/Algu } \\
\text { nas } \\
\text { veces }\end{array}$ & $\begin{array}{c}\text { 4. De } \\
\text { acuerdo } \\
I \\
\text { Casi } \\
\text { siempre }\end{array}$ \\
\hline \multirow{6}{*}{$\begin{array}{l}\text { 응 } \\
\text { 음 } \\
\text { 엉 }\end{array}$} & $\begin{array}{l}\text { 11. La diversidad constituye un } \\
\text { aspecto positivo y enriquecedor } \\
\text { para la danza }\end{array}$ & $4.07( \pm 1.21)$ & $7.9 \%$ & $5.1 \%$ & $7.9 \%$ & $29.8 \%$ \\
\hline & $\begin{array}{l}\text { 12. La diversidad del alumnado de } \\
\text { danza es común en nuestra } \\
\text { institución }\end{array}$ & $3.88( \pm 1.22)$ & $8.8 \%$ & $6.6 \%$ & $7.7 \%$ & $40.6 \%$ \\
\hline & $\begin{array}{l}\text { 13. La atención a la diversidad } \\
\text { ocupa un papel importante en la } \\
\text { práctica docente }\end{array}$ & $3.98( \pm 1.31)$ & $9.5 \%$ & $8.4 \%$ & $4.9 \%$ & $28.7 \%$ \\
\hline & $\begin{array}{l}\text { 14. La diversidad nos remite al } \\
\text { hecho de que el alumnado tiene } \\
\text { necesidades propias y específicas }\end{array}$ & $4.07( \pm 1.24)$ & $7.5 \%$ & $8.6 \%$ & $3.1 \%$ & $30.9 \%$ \\
\hline & $\begin{array}{l}\text { 15. La diversidad se constituye por } \\
\text { las diferencias físicas, de edad, } \\
\text { sexo, religión y otras }\end{array}$ & $4.04( \pm 1.30)$ & $9.3 \%$ & $8.2 \%$ & $3.1 \%$ & $27.6 \%$ \\
\hline & $\begin{array}{l}\text { 16. La diversidad física retrasa el } \\
\text { proceso formativo homogéneo }\end{array}$ & $3.70( \pm 1.27)$ & $7.7 \%$ & $16.1 \%$ & $5.5 \%$ & $39.5 \%$ \\
\hline \multirow{4}{*}{$\begin{array}{l}\frac{}{0} \\
\frac{0}{0} \\
\frac{0}{0} \\
\frac{0}{0}\end{array}$} & $\begin{array}{l}\text { 17. Estoy a favor de la inclusión en } \\
\text { danza, de la diversidad corporal }\end{array}$ & $3.68( \pm 1.35)$ & $11 \%$ & $11.3 \%$ & $12.8 \%$ & $27.8 \%$ \\
\hline & $\begin{array}{l}\text { 18. La carrera en danza debe } \\
\text { adaptarse a las nuevas } \\
\text { generaciones }\end{array}$ & $3.63( \pm 1.33)$ & $9.9 \%$ & $12.2 \%$ & $17.9 \%$ & $24.9 \%$ \\
\hline & $\begin{array}{l}\text { 19. No se deben admitir personas } \\
\text { con escasas cualidades físicas para } \\
\text { la danza }\end{array}$ & $3.84( \pm 1.28)$ & $7.1 \%$ & $13.2 \%$ & $9.1 \%$ & $28.9 \%$ \\
\hline & $\begin{array}{l}\text { 20. No sugiero la inclusión en } \\
\text { danza, un bailarín debe tener una } \\
\text { técnica y figura perfectas }\end{array}$ & $3.08( \pm 1.34)$ & $11.3 \%$ & $27.9 \%$ & $27.2 \%$ & $8.4 \%$ \\
\hline \multirow{6}{*}{ 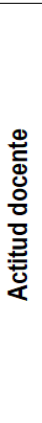 } & $\begin{array}{l}\text { 38. Los docentes motivan a los } \\
\text { estudiantes reforzando sus logros } \\
\text { 39. Los docentes facilitan la }\end{array}$ & $3.76( \pm 1.27)$ & $7.5 \%$ & $11.7 \%$ & $14.8 \%$ & $28.5 \%$ \\
\hline & $\begin{array}{l}\text { solución de conflictos mediante el } \\
\text { diálogo }\end{array}$ & $3.89( \pm 1.14)$ & $6.2 \%$ & $6.6 \%$ & $14.3 \%$ & $37.1 \%$ \\
\hline & $\begin{array}{l}\text { 40. Los docentes priorizan la } \\
\text { evaluación de los logros técnicos } \\
\text { corporales }\end{array}$ & $3.75( \pm 1.16)$ & $3.5 \%$ & $15.5 \%$ & $15.5 \%$ & $32.7 \%$ \\
\hline & $\begin{array}{l}\text { 41. Los docentes muestran un } \\
\text { comportamiento excluyente con } \\
\text { algunos estudiantes }\end{array}$ & $3.56( \pm 1.31)$ & $11.5 \%$ & $8.4 \%$ & $24.3 \%$ & $24.1 \%$ \\
\hline & $\begin{array}{l}\text { 42. Los docentes trabajan en } \\
\text { colaboración con otros colegas } \\
43 \text {. Los docentes promueven la }\end{array}$ & $3.76( \pm 1.27)$ & $7.5 \%$ & $11.7 \%$ & $14.8 \%$ & $28.5 \%$ \\
\hline & $\begin{array}{l}\text { coevaluación como estrategia para } \\
\text { sensibilizar a los estudiantes }\end{array}$ & $3.89( \pm 1.14)$ & $6.2 \%$ & $6.6 \%$ & $14.3 \%$ & $37.1 \%$ \\
\hline
\end{tabular}


Como se puede apreciar, los ítems $11,13,14$ y 15 que expresan sensibilidad hacia la inclusión, presentan en promedio las frecuencias porcentuales más altas $(79.15 \%)$, lo cual contrasta con la idea que tienen los estudiantes acerca de no admitir personas con escasas cualidades físicas (ítem 19: 70.6\%), y al mismo tiempo estar a favor de aceptar personas con diversidad anatómica, fisiológica e intelectual en esta carrera (ítem 17: 64.9\%). En los ítems 17 (64.9\%) y 19 (70.6\%) se denota una distribución semejante de respuestas a pesar de ser opuestas en su sentido, revelando que, aunque hay tendencias de opiniones favorables respecto a prácticas inclusivas, la respuesta a la propuesta radical de "no aceptar personas con escasas cualidades físicas" recibe un notable grado de aceptación. Esto es posible que responda a que la expresión "escasas cualidades físicas" puede ser asociada más a causas voluntarias por falta de empeño o cultivo de las cualidades por parte del sujeto, mientras que el tema de la inclusión y la diversidad denota, más bien, impedimentos congénitos, no voluntarios.

En la tabla 5, se muestra la medida con que el alumnado percibe el tratamiento que la institución y el currículo brinda al fortalecimiento de la atención a la diversidad.

Tabla 5.-Valoración sobre la postura de la institución y el currículo.

\begin{tabular}{|c|c|c|c|c|c|c|}
\hline & İtem & Media (d.e) & $\begin{array}{l}1 . \\
\text { Totalme } \\
\text { nte en } \\
\text { desacu } \\
\text { erdo/Nu } \\
\text { nca }\end{array}$ & $\begin{array}{l}\text { 2. En } \\
\text { desacu } \\
\text { erdo/Ca } \\
\text { si } \\
\text { nunca }\end{array}$ & $\begin{array}{l}\text { 3. No lo } \\
\text { sé/Algu } \\
\text { nas } \\
\text { veces }\end{array}$ & $\begin{array}{l}\text { 4. De } \\
\text { acuerdo } \\
\quad / \\
\text { Casi } \\
\text { siempre }\end{array}$ \\
\hline \multirow{5}{*}{ 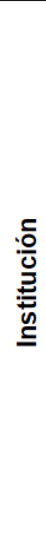 } & $\begin{array}{l}\text { 25. La institución ofrece } \\
\text { capacitación y apoyo } \\
\text { para reforzar la atención a la } \\
\text { diversidad }\end{array}$ & $3.83( \pm 1.34)$ & $11.7 \%$ & $8.6 \%$ & $4 \%$ & $35.8 \%$ \\
\hline & $\begin{array}{l}\text { 26. Las aulas están distribuidas } \\
\text { según las necesidades de cada } \\
\text { grupo }\end{array}$ & $3.29( \pm 1.37)$ & $12.6 \%$ & $23.6 \%$ & $8.2 \%$ & $33.3 \%$ \\
\hline & $\begin{array}{l}\text { 27. La institución promueve una } \\
\text { organización inclusiva, mediante } \\
\text { proyectos colaborativos }\end{array}$ & $3.51( \pm 1.36)$ & $13.5 \%$ & $13.9 \%$ & $7.1 \%$ & $39.1 \%$ \\
\hline & $\begin{array}{l}\text { 28. La institución promueve una } \\
\text { cultura de atención y respeto a la } \\
\text { diversidad }\end{array}$ & $3.81( \pm 1.31)$ & $10.4 \%$ & $8.8 \%$ & $8.4 \%$ & $34 \%$ \\
\hline & $\begin{array}{l}\text { 29. Las condiciones arquitectónicas } \\
\text { favorecen el acceso a todas las } \\
\text { áreas }\end{array}$ & $3.55( \pm 1.40)$ & $12.8 \%$ & $15.7 \%$ & $8.6 \%$ & $28.7 \%$ \\
\hline \multirow{4}{*}{$\frac{\circ}{\bar{J}}$} & $\begin{array}{l}\text { 34. El currículo es flexible, permite } \\
\text { la adaptación y el ajuste necesario } \\
\text { 35. Los criterios de selección para }\end{array}$ & $3.77( \pm 1.20)$ & $7.3 \%$ & $10.4 \%$ & $11.5 \%$ & $39.3 \%$ \\
\hline & $\begin{array}{l}\text { entrar a danza están regidos } \\
\text { principalmente por las condiciones } \\
\text { físicas }\end{array}$ & $3.73( \pm 1.32)$ & $10.2 \%$ & $13 \%$ & $5.1 \%$ & $37.1 \%$ \\
\hline & $\begin{array}{l}\text { 36. La licenciatura en danza permite } \\
\text { el acceso a todos los interesados en } \\
\text { el área }\end{array}$ & $3.74( \pm 1.34)$ & $11.3 \%$ & $10.6 \%$ & $7.5 \%$ & $33.8 \%$ \\
\hline & $\begin{array}{l}\text { 37. Los estudiantes con } \\
\text { necesidades específicas reciben la } \\
\text { misma clase que el resto de sus } \\
\text { compañeros }\end{array}$ & $3.49( \pm 1.28)$ & $10.2 \%$ & $15 \%$ & $14.1 \%$ & $36.9 \%$ \\
\hline
\end{tabular}


El aspecto mejor valorado por los estudiantes es el concerniente al apoyo y la capacitación que la institución ofrece en materia de apoyo a la diversidad (ítem 25: 75.8\%). Predomina también un porcentaje bastante alto en lo correspondiente a la promoción de una cultura de atención y respeto a la diversidad (ítem 28: $72.4 \%$ ), lo cual, se asemeja a la opinión en lo que respecta a los criterios de selección para entrar a la carrera que refieren estar mayormente enfocados a las condiciones físicas (ítem 35:71.8\%). Algo a resaltar, son los altos niveles de acuerdo en el último factor, relacionado con los aspectos curriculares de la institución, según lo entienden los estudiantes (ítems 34 a 37), y el notablemente más bajo nivel de acuerdo respecto a la atención que reciben los compañeros con necesidades específicas.

\section{2.-Prueba de Kruskal-Wallis: área de estudios.}

Con el propósito de conocer si el área de estudio a la que pertenecen los estudiantes (danza clásica [DC], danza contemporánea [DCo] y danza folklórica [DF]) establece diferencias en cuanto a la disposición que muestran hacia la diversidad, se recurrió a la revisión del comportamiento de las frecuencias porcentuales y se aplicó la prueba de hipótesis de Kruskal Wallis al factor 3, disposición hacia la diversidad, conformado por las variables 17, 18, 19 y 20 (Tablas 6 y 7). Cabe mencionar que los ítems 19 y 20 contienen proposiciones contrarias a la inclusión, por lo que los valores altos expresan posturas de desacuerdo con la inclusión y la atención a la diversidad. Se estableció como hipótesis de investigación que en cada uno de las cuatro variables aleatorias (postura favorable a la inclusión en la danza, necesidad de adaptación de la danza a las nuevas generaciones, rechazo a admitir personas con escasas cualidades físicas en la danza y desacuerdo con la inclusión de personas con condiciones diferentes en la danza) existen diferencias estadísticamente significativas asociadas al área dancística (variable de agrupación).

Tabla 6.-Prueba de Kruskal-Wallis para muestras independientes.

\begin{tabular}{llrrrr}
\hline Submuestra & & Ítem 17 & Ítem 18 & Ítem 19 & Ítem 20 \\
\hline Danza Clásica (Ballet) & Media & 1.9058 & 1.9493 & 3.8623 & 4.3696 \\
& N & 138 & 138 & 138 & 138 \\
& Desv. típ. & .79126 & .77669 & .81224 & .48445 \\
& Mediana & 2.0000 & 2.0000 & 4.0000 & 4.0000 \\
Danza Contemporánea & Media & 4.7887 & 4.7465 & 1.1127 & 1.7512 \\
& $\mathrm{~N}$ & 213 & 213 & 213 & 213 \\
& Desv. típ. & .40917 & .43605 & .31694 & .87365 \\
& Mediana & 5.0000 & 5.0000 & 1.0000 & 1.0000 \\
Danza Folklórica & Media & 3.7941 & 3.5784 & 2.0000 & 3.3824 \\
& Nesv. típ. & .40634 & .49625 & .00000 & .48836 \\
& Mediana & 4.0000 & 4.0000 & 2.0000 & 3.0000 \\
Total & Media & 3.6865 & 3.6313 & 2.1501 & 2.9161 \\
& N & 453 & 453 & 453 & 453 \\
& Desv. típ. & 1.35957 & 1.33323 & 1.28624 & 1.34757 \\
& Mediana & 4.0000 & 4.0000 & 2.0000 & 3.0000 \\
\hline & & Elaboración propia & & &
\end{tabular}

Tabla 7.-Resumen de prueba Kruskal-Wallis para muestras independientes.

\begin{tabular}{lllll}
\hline $\mathbf{N}$ total & $\mathbf{4 5 3}$ & & & \\
\hline & Item 17 & Ítem 18 & Ítem 19 & Ítem 20 \\
Estadístico de prueba & $379.672 ?$ & $372.743 ?$ & $400.206 ?$ & 351.332 \\
& & & & $?$ \\
Grados de libertad & 2 & 2 & 2 & 2 \\
Significación asintótica (prueba bilateral) & .000 & .000 & .000 & .000 \\
\hline
\end{tabular}


El estadístico de contraste Chi cuadrado de la prueba de Kruskal-Wallis, tiene asociado un valor menor $(S i g=0.00)$ que el nivel de significancia prefijado $(p=0.05)$, por tanto, la prueba sugiere aceptar las hipótesis de trabajo para cada ítem. Se puede asumir que la diferencia es significativa entre las distintas submuestras, siendo la submuestra de danza clásica donde hay menor disposición hacia la inclusión.

\section{4.-Conclusión.}

El presente estudio ha analizado la opinión de estudiantes de danza en cuatro universidades de México sobre un tema muy controversial en el campo de las artes: la inclusión y atención a la diversidad. Con base en los resultados obtenidos, se identifican aspectos muy importantes que dan respuesta a las metas de este estudio. Se pudo diagnosticar la disposición general del alumnado de danza hacia la atención a la diversidad e inclusión y, desde la perspectiva personal de cada uno, se pudieron identificar las actuaciones y posturas más recurrentes de los docentes e instituciones ante esta temática, así como determinar si el área dancística de formación incide en las actitudes hacia la diversidad. Así pues, en primer lugar, hay elementos para considerar que existen algunas contradicciones en el hecho de que las frecuencias porcentuales sean más altas en algunos ítems que reflejan sensibilidad hacia la diversidad, mientras en otros también sensibles reflejan la actitud contraria; entre otras situaciones, estar a favor de la inclusión y de aceptar personas con diversidad corporal, pero al mismo tiempo estar en desacuerdo de la inclusión de personas con escasas cualidades físicas para la danza. Por otro lado, se pudo percibir que los estudiantes de danza poseen un alto grado de conocimiento acerca de los concepto de diversidad e inclusión, lo que estos implican para la danza y el lugar que debería tener dentro de la práctica cotidiana; sin embargo, a pesar de ser un gran número de encuestados los que asumen con disposición el tema, otro tanto apoya la idea de que las diferentes cualidades físicas y habilidades que presentan algunos estudiantes son un factor que puede retrasar el proceso de enseñanza y aprendizaje del resto del grupo. Asimismo, gran parte de ellos considera necesario que la danza se adapte a las nuevas generaciones, es decir, a los nuevos cuerpos, pensamientos y necesidades de cada individuo, pero no sugieren del todo la inclusión de personas en condiciones de diversidad dentro de la danza profesional, ya que, en proporciones prácticamente equivalentes, unos aseguran que el bailarín debe mantener una impecable calidad técnica y una figura corporal perfecta, otros no tienen clara una decisión absoluta y otros más están en desacuerdo totalmente. En segundo lugar, sobresale el hecho de centrar la atención a la búsqueda de la perfección técnica corporal, lo que refuerza una importante tendencia a la práctica tradicional. Asimismo, desde la perspectiva del alumnado, prevalece en los docentes un comportamiento excluyente poco ajustado a la práctica de una cátedra inclusiva, que sin duda requiere de la capacidad de respetar las características propias de cada persona y comprender que las habilidades de cada una son diferentes (Males \& Díaz, 2017). Lamentablemente, la construcción de políticas que pretenden combatir la discriminación para impulsar la tolerancia y el respeto, terminan siendo una obra de teatro donde se actúa con la lógica del disimulo, es decir, se continúa discriminando, pero se oculta. De nada sirven los objetivos propuestos para el desarrollo sostenible si sólo se advierten en textos o exposiciones llenos de tinta o discurso; 
es necesaria la práctica sensata y honesta. Por otra parte, una tercera conclusión es que se requiere fomentar el uso de instrumentos de evaluación en el área de la danza. Por ello, sería conveniente promover las ventajas que ofrece el enfoque socioformativo en los procesos de evaluación para la construcción de rúbricas socioformativas en atención a las necesidades propias de la danza, y lograr al igual que otros estudios como los de Loera-Ramírez y ParraAcosta (2017); y Rodríguez y Caiza (2019), promover el desarrollo de profesionales competentes, con valores comunitarios, reflexivos, comprometidos y comprensivos. Una cuarta situación, es que en la población estudiantil encuestada existe mucha confusión e indecisión sobre si apoyar o no la diversidad. Hay una fuerte tendencia a rechazar la diversidad en danza y latentes dudas al respecto, sobre todo en el área específica de la danza clásica, seguida por el área de danza contemporánea y folklor. Esto resalta la idea de que los diversos motivos, oportunidades, necesidades y expresiones existentes de la diversidad en estudiantes universitarios pueden ser una oportunidad para estimular la innovación educativa en el área de la danza, y dejar de pensar en el individuo ideal que niega la existencia de la diversidad con una pretensión homogeneizadora. Se manifiesta lo complejo que resulta brindar atención a la diversidad en el área de la danza, ya que priman criterios muy particulares para su desarrollo, entre los cuales, la perfección corporal suele revestir una importancia substancial. Por lo tanto, la educación inclusiva que busca responder a las diferentes necesidades del alumnado como prioridad, contrapone la estructura tradicional con la que se han formado miles de generaciones de bailarines, pues es difícil imaginar a un grupo de bailarinas en escena sin formas virtuosas y perfectas.

Con estos primeros resultados, se observa un gran esfuerzo por desarrollar competencias orientadas a la atención de la diversidad. Aunque las exigencias institucionales demanden fines políticos que no siempre advierten apoyo y asesoría para fortalecer el entorno, y los docentes recurran a la práctica tradicional por inercia, por desconocer otras alternativas o por resistencia a la innovación, existe una gran responsabilidad profesional que, hoy en día, no puede ser soslayada. Es momento de que los procesos formativos en danza comiencen a promover una práctica inclusiva, generar espacios incluyentes que reúnan grupos heterogéneos, promover la sensibilización, y enriquecer el aprendizaje a partir de las propias diferencias y la igualdad de oportunidades. Cumplir con esta importante misión hacia la atención a la diversidad en danza requiere de constantes esfuerzos. Se requiere la disposición de todos los protagonistas del mundo dancístico, especialmente el sector más joven, que es el que puede ir empujando los cambios desde abajo y asimismo permitir buscar soluciones y cambios progresivos que ayuden a modificar la cultura de las universidades mexicanas hacia un desarrollo serio de las prácticas inclusivas. Seguramente, la capacidad de querer conseguirlo está presente, pero la energía destinada para que esa capacidad se desarrolle no llegará sola; se requieren mecanismos tangibles que pongan en marcha su funcionalidad (Bartolomé, 2017).

Por otro lado, se considera que la implementación de modificaciones curriculares o metodológicas en los programas de estudio no es suficiente, se deben implementar procesos de investigación que promuevan el desarrollo humano, la construcción de instrumentos evaluativos que sintonicen con las realidades del contexto y sobre todo, que ofrezcan insumos para tomar decisiones reales; sería importante conocer lo que la sociedad opina sobre la diversidad en danza, pues finalmente los bailarines se entregan al público, expresan en 
escena la vida social y cultural de todo un pueblo, y esperan los aplausos del público como premio a sus esfuerzos. Por ello, sería interesante saber la disposición que tiene la sociedad en aceptar la diversidad, saber si están dispuestos a entender la complexión diversa y cualidades técnicas no tan perfectas en la danza; realmente ¿disfrutarían y consumirían de igual forma una danza en la diversidad?, en definitiva, la inclusión educativa no es una meta sino un proceso de cambio, una nueva forma de ser, estar y hacer.

Finalmente, se sugiere la aplicación del instrumento en diversas instituciones, o bien el diseño de instrumentos que permitan la autoevaluación y sensibilización para la búsqueda de formas creativas e innovadoras de implementación de la inclusión de personas en situación de diversidad en la formación dancística profesional, que sostenga simultáneamente la calidad de las ejecuciones y el desarrollo del talento de los profesionales a partir de sus propias condiciones personales.

\section{5.-Referencias.}

Avila García, Y., y Veytia Bucheli, M. (2019). Análisis documental sobre los procesos de enseñanza/aprendizaje de la danza. Educación y Humanismo, 21(37), 67-85. https://doi.org/10.17081/eduhum.21.37.3410

Bartolomé, M. (2017). Diversidad educativa ¿Un potencial desconocido? Revista de Investigación Educativa, 35(1), 15-33. http://dx.doi.org/10.6018/rie.35.1.275031

Campo, L., Palys, A. M., Passo, P., y Tamayo S. (2018). Diálogos en movimiento: Reflexiones teórico-metodológicas de la danza como recurso de investigación interdisciplinar. Barcelona, España: GRAFO working papers, 7, 1-37. https://doi.org/10.5565/rev/grafowp.32

Cornejo, C. (2017). Respuesta educativa en la atención a la diversidad desde la perspectiva de profesionales de apoyo. Revista Colombiana de Educación, (73), 7594. Recuperado de: https://www.redalyc.org/pdf/4136/413651843005.pdf

Corrales, A., Mena, M., García, J. J., y López Liria, R. (2017). Prevención de las principales lesiones en la danza y mecanismos de producción. INFAD, Revista de Psicología, 2(1), 239-248. https://doi.org/10.17060/ijodaep.2017.n1.v2.936

Loera Ramírez, M. O., y Parra Acosta, H. (2017). Aprendizaje de la enfermedad pulmonar obstructiva crónica utilizando Aprendizaje Basado en Problemas. Un estudio comparativo. Revista de Educación y Desarrollo, 40, 61-65. Recuperado de: http://www.cucs.udg.mx/revistas/edu_desarrollo/anteriores/40/40_Loera.pdf

Males, V. H., y Díaz, A. (2017). Estrategias de enseñanza, aprendizaje y evaluación inmersas en el currículo oculto en las prácticas de formación de docentes en los grupos artísticos de danzas folclóricas a nivel universitario. Biumar, 1(1), 156-162. Recuperado de: http://ito.mx/Ljjb 
Rodríguez, J., y Caiza, M. (2019). Formación de gestores comunicacionales de salud comunitaria mediante integración universitaria. Revista Chakiñan de Ciencias Sociales y Humanidades, (7), 84-93. Recuperado de: https://bit.ly/2LuEqUC

Tobón, S. (2017). Ejes esenciales de la sociedad del conocimiento y la socioformación. Mount Dora: Kresearch. Recuperado de: https://bit.ly/2Cc1KA5

UNESCO (2016). Educación 2030: Declaración de Incheon y Marco de Acción para la realización del Objetivo de Desarrollo Sostenible 4: Garantizar una educación inclusiva y equitativa de calidad y promover oportunidades de aprendizaje permanente para todos. (página web). Recuperado de: https://unesdoc.unesco.org/ark:/48223/pf0000245656_spa/PDF/245656spa.pdf.mu Iti 